\title{
ON CHARACTERISTIC CLASSES OF FLAT RIEMANNIAN MANIFOLDS
}

\author{
BY A. T. VASQUEZ
}

\author{
Communicated by L. Auslander, February 21, 1968
}

The "geometric" subject of compact, connected, flat Riemannian manifolds has long ago been "reduced" to purely algebraic questions. Such manifolds are determined (up to a connexion preserving diffeomorphism) by the isomorphism class of their fundamental groups. A precise characterization of those groups which arise in this way is also classical. See [4] or [8] for a convenient modern account. Despite this, apparently simple geometric questions remain unanswered. One such which has intrigued the author and others is this: does every compact flat Riemannian manifold bound a manifold of one higher dimension? If not, which elements of the cobordism ring can be represented by such manifolds? It is known [6], [7], of course, that this question turns on the values of the Stiefel-Whitney numbers and, in the orientable case, the Pontrjagin numbers of the manifold.

A few general facts are easy to establish. Since the structure group of the tangent bundle of any Riemannian manifold is reducible to its holonomy group and since the holonomy group of a compact connected flat Riemannian manifold is finite, the Pontrjagin classes are all torsion classes. It follows that the Pontrjagin numbers vanish (orientability, of course, must be assumed for the statement even to make sense). Thus, such a manifold determines a torsion element in the oriented cobordism ring. A similar argument shows that if the order of the holonomy group is odd, the Stiefel-Whitney classes vanish. Thus, in this case the manifold automatically bounds, and if it is orientable, bounds an orientable manifold. Such optimism that these simple arguments may arouse is restrained by the example [1] of Auslander and Szczarba showing that the Stiefel-Whitney classes themselves do not always vanish.

In view of the sharp dependence on the holonomy group, it seems reasonable to consider for each finite group, $\Phi$, the class of compact connected flat Riemannian manifolds having holonomy group isomorphic to $\Phi \ldots \Phi$ - . manifolds for short.

Recall that the tangent bundle of any differentiable manifold, $X$, determines the characteristic subalgebra of the cohomology algebra, $H^{*}(X ; R)$. Here $R$ is any ring of coefficients; when $R$ is the integers modulo two, the characteristic subalgebra is generated by the StiefelWhitney classes. One geometric consequence of the algebraic theorem 
below is this: one can assign an integer, $n(\Phi)$, to each finite group $\Phi$ such that the characteristic algebra of any $\Phi$-manifold is zero in dimensions greater than $n(\Phi)$. In particular, if the dimension of the $\Phi$-manifold exceeds $n(\Phi)$, it necessarily bounds. It follows that for fixed $\Phi$ only finitely many $\Phi$-manifolds can fail to bound.

A mild variant of the main theorem was conjectured by L. Auslander. I wish to thank him for framing the problem in this way as well as for many useful and stimulating conversations on this and related topics.

Notation. A Bieberbach group is one having a normal subgroup of finite index which is a finitely generated abelian group. The groups which arise as fundamental groups of compact connected flat Riemannian manifolds are precisely the torsion-free Bieberbach groups. In such a group, those elements having only finitely many conjugates make up a normal subgroup of the prescribed type and (for geometric reasons) is called the translation subgroup. Its rank is the dimension of the group (again the terminology has geometric motivation although it is indeed the homological dimension of the group). The corresponding (finite) quotient group is the holonomy group.

MAIN TheOREM. For each finite group, $\Phi$, there is an integer $n(\Phi)$ such that: if $\pi$ is a torsion-free Bieberbach group with holonomy group isomorphic to $\Phi$, then the translation subgroup of $\pi$ contains a normal subgroup, $N$, such that $\pi / N$ is again a torsion-free Bieberbach group and dimension $(\pi / N) \leqq n(\Phi)$.

Corollary 1. Suppose $X$ is a $\Phi$-manifold and dimension $X>n(\Phi)$. Then $X$ fibres over another compact connected flat Riemannian manifold having dimension $\leqq n(\Phi)$. Furthermore the fibre is a torus and the tangent bundle of $X$ is induced from a bundle over the base manifold.

Corollary 2. If $X$ is a $\Phi$-manifold, its characteristic algebra in dimensions greater than $n(\Phi)$ is zero.

Corollary 3. If $X$ is a $\Phi$-manifold and $\operatorname{dim} X>n(\Phi)$, then $X$ bounds a manifold of one higher dimension.

CoROLLARY 4. For each finite group $\Phi$ at most finitely many $\Phi-m a n i-$ folds can fail to bound.

We remark that a relatively simple argument concerning StiefelWhitney numbers shows that whether a $\Phi$-manifold bounds or not is equivalent to asking the same question about a certain $\Phi_{2}$-manifold where $\Phi_{2}=$ the 2-Sylow subgroup of $\Phi$. Corollary 3 can be "improved" 
by replacing "dim $X>n(\Phi)$ " by “dim $X>\min \left(n(\Phi), n\left(\Phi_{2}\right)\right)$." So little is known about $n(\Phi)$, however, that this seems a futile exercise. Indeed, the only concrete fact known is that $n(Z / 2 Z)=1$ and $n(Z / 2 Z \oplus Z / 2 Z) \leqq 6$.

The proof of the theorem uses the homological machinery introduced in [2]. In that language, one is given a finite group $\Phi$, a $\Phi$ module $M$, and a special point $\alpha \in H^{2}(\Phi ; M)$; one wants then to show that $M$ contains a pure submodule, $N$, such that rank $M / N \leqq n(\Phi)$ and $M \rightarrow M / N$ takes $\alpha$ to a special point of $H^{2}(\Phi ; M / N)$. Actually the results of [5] were exploited in [3], to handle the case in which $\Phi$ is a group of prime order. (The point of view and language of [3] are wholly different however.) It is essentially elementary to pass from that special case to the general-the main difficulty being a suitable definition of $n(\Phi)$.

\section{REFERENCES}

1. L. Auslander and R. H. Szczarba, Characteristic classes of compact solvmanifolds, Ann. of Math. (2) 76 (1962), 1-8.

2. L. Charlap, Compact flat Riemannian manifolds. I, Ann. of Math. (2) 81 (1965), 15.

3. L. Charlap, and A. T. Vasquez, Compact flat Riemannian manifolds. II, Amer. Math. 87 (1965), J. 551-563.

4. S. Kobayashi and K. Nomizu, Foundations of differential geometry, Vol. 1, Wiley, New York, 1963.

5. I. Reiner, Integral representations of cyclic groups of prime order, Proc. Amer. Math. Soc. 8 (1957), 142.

6. R. Thom, Quelques propriêtes globales des varietés differentiables, Comment. Math. Helv. 28 (1954), 17-86.

7. C. T. C. Wall, Determination of the cobordism ring, Ann. of Math. (2) 72 (1960), 292-311.

8. J. A. Wolf, Spaces of constant curvature, McGraw-Hill, New York, 1967.

City University of New York 